\title{
Analysis of MR Images of Mice in Preclinical Treatment Monitoring of Polycystic Kidney Disease
}

\author{
Stathis Hadjidemetriou ${ }^{1}$, Wilfried Reichardt ${ }^{1}$, Martin Buechert ${ }^{2}$, \\ Juergen Hennig ${ }^{1}$, and Dominik von Elverfeldt ${ }^{1}$ \\ 1 University Hospital Freiburg, Dept. of Diagnostic Radiology, Medical Physics, \\ Hugstetter Street 55, 79106 Freiburg, Germany \\ efstathios.hadjidemetriou@uniklinik-freiburg.de \\ 2 Magnetic Resonance Development and Application Center, \\ Hugstetter Street 55, 79106 Freiburg, Germany
}

\begin{abstract}
A common cause of kidney failure is autosomal dominant polycystic kidney disease (ADPKD). It is characterized by the growth of cysts in the kidneys and hence the growth of the entire kidneys with eventual failure in most cases by age 50. No preventive treatment for this condition is available. Preclinical drug treatment studies use an in vivo mouse model of the condition. The analysis of mice imaging data for such studies typically requires extensive manual interaction, which is subjective and not reproducible. In this work both untreated and treated mice have been imaged with a high field, 9.4T, MRI animal scanner and a reliable algorithm for the automated segmentation of the mouse kidneys has been developed. The algorithm first detects the region of interest (ROI) in the image surrounding the kidneys. A parameterized geometric shape for a kidney is registered to the ROI of each kidney. The registered shapes are incorporated as priors to the graph cuts algorithm used to extract the kidneys. The accuracy of the automated segmentation has been demonstrated by comparing it with a manual segmentation. The processing results are also consistent with the literature for previous techniques.
\end{abstract}

\section{Introduction}

In autosomal dominant polycystic kidney disease (ADPKD), cysts progressively enlarge and accumulate fluid as well as possibly blood. The cysts disrupt the renal parenchyma and increase the total volume of the kidneys significantly. This eventually leads to end stage renal disease in most cases by age 50. No approved treatment for this condition is available, although clinical trials are performed with compounds known to slow its progression [1. Early stages of ADPKD are asymptomatic, which has made the assessment of disease progression more difficult and has also hindered the development of medical treatments. The early kidney enlargement, however, associated with the disease progression can be assessed with imaging. 
Preclinical drug treatment studies involve models of ADPKD in mice. The imaging of mouse kidneys has been performed in vivo primarily with X-ray micro-CT [2] and MRI 345]. Typically, the analysis of the images has been performed with extensive manual annotation 45]. This is time consuming due to the large number of mice involved in preclinical trials, subjective, and not reproducible. There have been attempts to restrict the manual interaction to the initialization of an automated segmentation. In CT images axial contours of kidneys were represented with a deformable model [2]. In MRI the largest coronal contours of kidneys were represented with splines or as the shortest path between annotated points [3]. MRI allows the repeated and accurate imaging of the kidneys without the use of a contrast agent, and thus the possibility of additional kidney complications. More generally, images of healthy human kidneys from MRI and CT have been processed with a variety of algorithms such as level sets [6] and graph cuts [7, often combined with a shape prior. A sufficiently validated and automated method for the volumetric quantification of polycystic kidneys from MR images and for the monitoring of treatment has not been evident in the literature.

In this work mouse models of ADPKD have been imaged with a high resolution MRI animal scanner. The image region of interest (ROI) surrounding the kidneys is localized and processed to restore intensity uniformity. Subsequently, a prior superspheroid shape 8 is registered to the ROI of each kidney. The registered shape is incorporated into the graph cuts algorithm, which provides the kidneys [9]. The automated processing has analyzed images of a preclinical trial for two compounds. The accuracy of the automated segmentation has been demonstrated by comparing it with a manual one by a medical expert. The processing results are also in agreement with those obtained with previous more interactive or invasive techniques, that these compounds slow the progression of ADPKD in rodent models [1].

\section{Methods}

\subsection{Mouse Model of ADPKD, Treatment, and Image Acquisition}

Twelve female mice bearing the $p c y / p c y$ genotype were used as a model of ADPKD. All experiments were performed in accordance with the local animal care commission. The mice were distributed into three groups of four. The first group was treated with intraperitonial application of $5 \mathrm{mg} / \mathrm{kg} / \mathrm{day}$ of rapamycin. A similar treatment was applied to a second group with vasopressin-2receptor antagonist SR121463 (Sanofi-Aventis). Both of these compounds have been shown to slow the rate of cystogenesis in mice and are currently on clinical trials [1. The third group was untreated and was used as control. The imaging began four weeks after the initiation of the treatment and was repeated every two weeks for a total of four time points.

The imaging was performed with a 9.4Tesla small bore MRI animal scanner, Bruker Biospin. The coil was a cylindrical quadrature birdcage resonator with 
an inner diameter of $38 \mathrm{~mm}$. To reduce motion artifacts the mice were anesthetized and the scan was performed with cardiac gating to also reduce blood flow artifacts. The acquisition was a fluid-sensitive T2-weighted spin echo RARE sequence $\left(T R / T E_{\text {eff }} / F A: 3000 \mathrm{~ms} / 36 \mathrm{~ms} / 180^{\circ}\right)$. The mice were placed horizontally on the $x-y$ plane along the coil axis, $y$. The field of view was coronal of $30 \times 30 \mathrm{~mm}^{2}$ with a matrix size of $256 \times 256$ and a resolution of $0.12 \times 0.12 \mathrm{~mm}^{2}$. The slice thickness $\Delta z$ was $0.5 \mathrm{~mm}$ without slice spacing. A sufficient number of slices, on average twenty-five, were included to ensure a complete coverage of both kidneys. The acquisition provided image $I(\mathbf{x}) \rightarrow \Re$, where $\mathbf{x}=(x, y, z)$. The middle coronal slices of four representative images are shown in the left column of figure 1 .

\subsection{Localization of the Image Region Surrounding the Kidneys}

The images are first processed to extract the foreground. The contribution of the noise in the background to the histogram $\mathcal{H}$ is a Rayleigh distribution $\mathcal{R}$ with low signal to noise ratio [10. It is fitted with its intensity of maximum density as well as its full width half maximum and subtracted from the image histogram to give $\mathcal{H}-\mathcal{R}$. The highest intensity, $i$, for which $\mathcal{R}(i)>\mathcal{H}(i)-\mathcal{R}(i)$ provides the upper bound of the intensity range of the background. That range is backprojected to the image and the corresponding voxels are set to zero. The largest connected component over the remaining non-zero image provides the foreground $I_{f}$.

The foregroung image in form $I_{f, b}=\left(I_{f}>0\right)$ provides a binary representation of the mouse body. The eigenvector $\mathbf{e}$ in $I_{f, b}(\mathbf{x})$ corresponding to the largest eigenvalue passes from the spatial mean of $I_{f, b}, \mathcal{O}$, along direction $\left(\theta_{e}, \phi_{e}\right)$, where $\theta_{e}$ is the rotation angle on the $x-y$ plane with the $x$ axis, and $\phi_{e}$ is the rotation angle with the $z$ axis. The eigenvector e together with vector $(0,0,1)$ provide the midsagittal plane, which lies approximately between the two kidneys. The image $I_{f}$ is also processed with a uniform spherical filter of radius $\rho$ centered on the midsagittal plane, where $\rho$ is the kidney's axial radius. The point on that plane with maximum response is the point between the two kidneys, $\mu$.

The shape of the kidneys is represented geometrically with a superspheroid, $f(\mathbf{x})=\left|\sqrt{\left(x^{2}+z^{2}\right) / a^{2}}\right|^{n}+|y / b|^{n}-1[8]$. The shape axis $y$ is initialized parallel to the eigenvector $\mathbf{e}$, with rotation $R\left(\theta_{e}, \phi_{e}\right)$. It is also initialized with $\rho=a<b$ to give a prolate superspheroid elongated along the mouse body axis. The exponent is set to $n=2.25$ to represent the flat anterior and posterior ends of the kidneys compared to those of a prolate spheroid. The next step is a rough estimation of the center points of the two kidneys $c_{s}^{\prime}$, where $s$ stands for the side that can be left, $c_{l}^{\prime}$, or right, $c_{r}^{\prime}$. They are initially set to distance $\rho$ from $\mu$ normal to the midsagittal plane. The location of each is varied to maximize $\sum I_{f}(\mathbf{x})$ in their interior $\{\mathbf{x}: f(\mathbf{x})<0\}$. This is performed with gradient descent and gives an improved estimate of the kidney centers, $c_{l}$ and $c_{r}$.

Subsequently, the fit of the prior shape is refined for each kidney by varying four $\mathbf{m}=\left(m_{1}, m_{2}, m_{3}, m_{4}\right)$ of the parameters of the geometric shape. The first, $m_{1}$, is an overall scale that affects both $a$ and $b$ identically. The second, $m_{2}$, 
affects the eccentricity $b / a$ along the body axis $y$. The third, $m_{3}$, is the $z$ coordinate of the center of the superspheroid. The last, $m_{4}$, is a rotation of angle $\theta$, $R_{(\theta, 0)}$, on the $x-y$ plane around the spatial mean of $I_{f, b}, \mathcal{O}$. The superspheroid instances during registration are given by:

$$
f\left(\mathbf{x}^{\prime}, \mathbf{m}\right)=\left|\sqrt{\frac{x^{\prime 2}+\left(z^{\prime}-m_{3}\right)^{2}}{m_{1}^{2} a^{2}}}\right|^{n}+\left|\frac{y^{\prime}}{m_{1} m_{2} b}\right|^{n}-1,
$$

where $n=2.25$, and $\left(x^{\prime}, y^{\prime}, z^{\prime}\right)=R_{\left(\theta_{e}+m_{4}, \phi_{e}\right)}(x, y, z)$. The shape provided by equation (1) is converted to a binary image, $I_{\text {shape } \mathbf{m}}(\mathbf{x})=(f(\mathbf{x}, \mathbf{m})<0)$. The union of the spheres centered around $c_{l}$, and $c_{r}$, each of radius $4 \rho$ provides the region of interest, $I_{R O I}$, which is further processed for the extraction of the kidneys. Some examples of $I_{R O I}$ are shown in the second column of figure 1 The fitting is performed over the ROI of $I_{f, b}(\mathbf{x})$ in the side around the midsagittal plane in which the kidney to be modeled lies, $I_{R O I, b, s}(\mathbf{x})=I_{f, b}(\mathbf{x}) \times I_{R O I}(\mathbf{x}) \times$ $I_{s}(\mathbf{x})$, where $\times$ is voxelwise multiplication and $s=\{l, r\}$.

The cost function for the shape fitting is the $L_{1}$ or equivalently the $L_{2}$ norm of the difference between the binary shape image $I_{\text {shape } \mathbf{m}}$ and the binary ROI for each kidney $I_{R O I, b, s}, \min _{\mathbf{m}}\left\|I_{\text {shape,m }}\left(\mathbf{x}-c_{s}\right)-I_{R O I, b, s}(\mathbf{x})\right\|_{1}$. The minimization of the cost is performed with gradient descent. The intermediate coronal slices of the ROI of four representative images and the computed kidney shapes are shown in the second and third columns of figure 1, respectively.

\subsection{Segmentation of the Kidneys from an Image}

The region of interest $I_{R O I}$ is processed for kidney segmentation. It is denoised with median filtering and processed to restore intensity uniformity [1]. Subsequently, the isotropic self-co-occurrence statistics of radius $u$ in the region of interest are analyzed with Otsu's algorithm to obtain an intensity $\tau$. The voxels in intensity range $\left[0, \alpha_{1} \tau\right]$, where $0<\alpha_{1}<1$ are set to background seeds and the voxels in intensity range $\left[\alpha_{2} \tau, \max _{\mathbf{x}} I(\mathbf{x})\right]$, where $1<\alpha_{2}$ are set to foreground seeds. The classification of the voxels with intensities in the intermediate range $\left(\alpha_{1} \tau, \alpha_{2} \tau\right)$ is ambiguous. The registered prior kidney shape cancels foreground seeds for which $I_{\text {shape } \mathbf{m}}(\mathbf{x})=0$ and sets their classification to ambiguous.

The ambiguities are resolved locally with the graph cuts algorithm $[9$. The image is represented as an undirected and weighted graph $\mathcal{G}(\mathcal{V}, \mathcal{E})$. 9]. The image voxels together with two bounding nodes $s$ and $t$ are the nodes $\mathcal{V}$ of the graph. A neighborhood edge $\{p, q\}$ is included in the graph between the node corresponding to every voxel $p$ and the node corresponding to voxel $q$ if $q \in \mathcal{N}_{p}$, where $\mathcal{N}_{p}$ is the 26-connected neighborhood of node $p$. Boundary edges are established from every node $p$ to both $s$ and $t$ to give $\{p, s\}$ and $\{p, t\}$. The edges are assigned weights, which simulate flow capacities $[9$. A subset of edges $\mathcal{C} \in \mathcal{E}$ is called an $s / t$-cut if the bounding nodes are completely separated in the induced graph $\mathcal{G}_{\mathcal{C}}=(\mathcal{V}, \mathcal{E}-\mathcal{C})$. The bounding node in $\mathcal{G}_{\mathcal{C}}$ to which a node $p$ remains connected to determines its classification as foreground if $\{p, s\} \in \mathcal{G}_{\mathcal{C}}$ and as background if $\{p, t\} \in \mathcal{G}_{\mathcal{C}}$. The $\operatorname{cost}|\mathcal{C}|$ of a cut is the sum of all edge weights in $\mathcal{C}$. The cut of globally minimum cost is provided by the equivalent maximum flow solution from $s$ to $t$. 

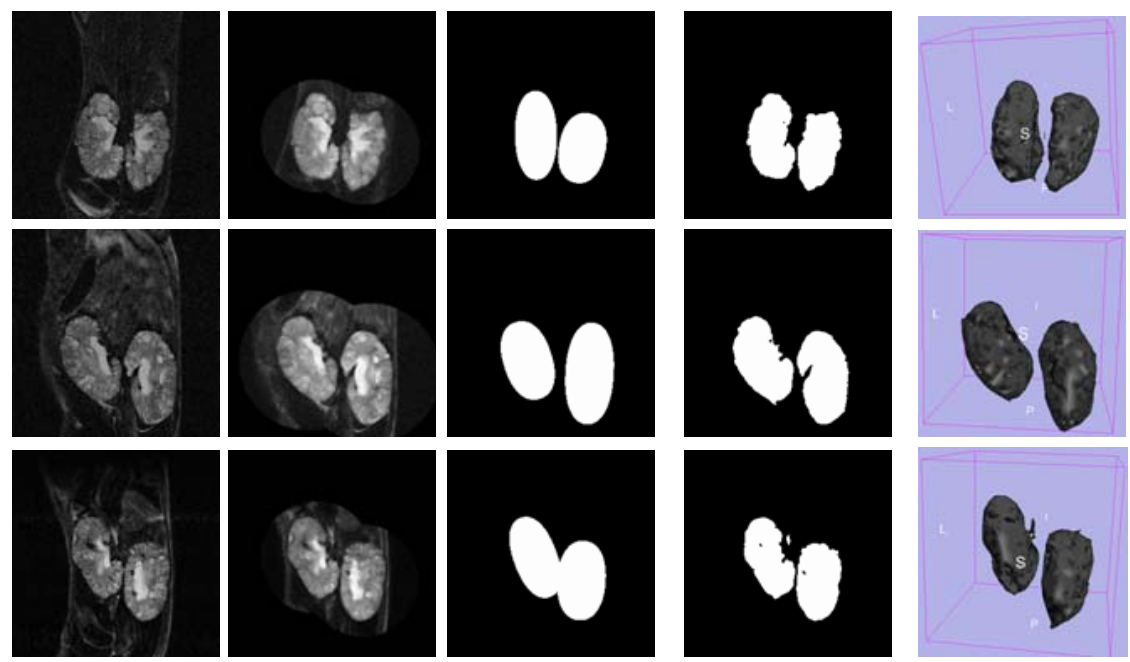

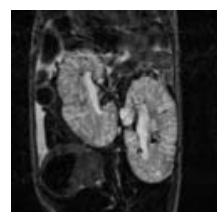

(a) Original

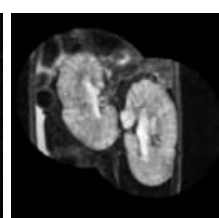

(b) ROI

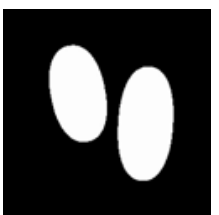

(c) Prior shape

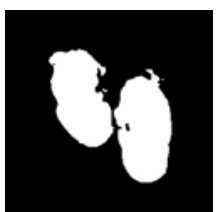

(d) Segmentation

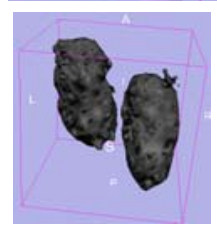

(e) Surface

Fig. 1. The processing of four representative images shown along the rows. The middle coronal slices of the original images are in the left, next to them are the regions of interest, in the third column are the prior shapes, and in the fourth column are the segmented kidneys. In the fifth column is a rendering of the segmentation surfaces.

The edge weights are positive. The total weight of neighborhood edge $\{p, q\} \in$ $\mathcal{N}, V_{T, p q}$, is a product of factors that depend on $I_{R O I}$ and $I_{\text {shape }}$. The weight derived from $I_{R O I}$ depends on the intensity difference between adjacent voxels $V_{1, p q}=\exp \left(-\left(I_{R O I}\left(\mathbf{x}_{p}\right)-I_{R O I}\left(\mathbf{x}_{q}\right)\right)^{2} / \sigma^{2}\right)$, where $\sigma$ is a fraction $\beta$ of the dynamic range, $\sigma=\beta\left(\max _{\mathbf{x}} I_{R O I}(\mathbf{x})-\min _{\mathbf{x}} I_{R O I}(\mathbf{x})\right)$. The anisotropic spatial resolution is represented by factor $V_{2, p q}=1 / d_{p q}$, where $d_{p q}=\left\|\mathbf{x}_{p}-\mathbf{x}_{q}\right\|_{2}$. The shape boundary provided by $\nabla I_{\text {shape }}$ is smoothed with a Gaussian filter of standard deviation equal to $\rho$. Subsequently, its dynamic range is rescaled with $\gamma$ and reversed to give $\Phi(\mathbf{x})=1-\gamma \nabla I_{\text {shape }}(\mathbf{x}) * G(\rho)$ as well as the shape edge weight $V_{3, p q}=\left(\Phi\left(\mathbf{x}_{p}\right)+\Phi\left(\mathbf{x}_{q}\right)\right) / 2$ [12]. The product:

$$
V_{T, p q}=\exp \left(-\frac{\left(I_{R O I}\left(\mathbf{x}_{p}\right)-I_{R O I}\left(\mathbf{x}_{q}\right)\right)^{2}}{\sigma^{2}}\right)\left(\frac{1}{d_{p q}}\right)\left(\frac{\Phi\left(\mathbf{x}_{p}\right)+\Phi\left(\mathbf{x}_{q}\right)}{2}\right)
$$

gives the total weight of edge $\{p, q\} \in \mathcal{N}$.

The weights to the bounding nodes are selected so that the minimum $s / t$ cut preserves the classification of the seed nodes as well as includes neighborhood edges and thus segments the image. Some representative images and the 
corresponding segmentations are shown in the second and fourth columns of figure 1. respectively. A surface rendering of the kidney segmentations is shown in the fifth column of figure 1

\section{Results: Data Analysis and Evaluation}

The implementation is in $C++$ and uses the ITK library [13. The radius of the self-co-occurrences was $u=3 \mathrm{~mm}$. The intensity range that provided the background seeds was $[0,0.3 \tau]$ and the intensity range that provided the foreground seeds was $\left[1.2 \tau, \max _{\mathbf{x}} I_{R O I}(\mathbf{x})\right]$, that is, $\alpha_{1}=0.3$ and $\alpha_{2}=1.2$. A value of $\beta=1 / 32$ was used for $\sigma$ in $V_{1, p q}$ and the value of $\gamma$ enforced the dynamic range of $V_{3, p q}$ to be $[0.7,1.0]$. These parameters were kept constant for the processing of all 48 images. Only the axial kidney radius $\rho$ varied with the input image. It is sufficient to select an approximate value for it by observation.

The automatic segmentations were compared with the region enclosed within slicewise manual outlinings of the boundaries of the kidneys for thirty images of both treated and untreated mice out of the total of 48 images analyzed. The manual outlinings were performed by a medical expert (M.D.), who is a co-author, blinded to the automated segmentations of the kidney regions. In the comparison an image is classified as kidney volume correctly detected, $T P$, kidney volume missed by the detection, $F N$, and volume falsely detected, $F P$. These values provide the recall $R E=\frac{T P}{T P+F N}$, and the precision $P R=\frac{T P}{T P+F P}$. A score of 1.0 for recall indicates completeness and a score of 1.0 for precision indicates exactness. The average and standard deviation of the precision were $P R=94.1 \pm 1.5 \%$, and those of the recall were $R E=94.6 \pm 1.8 \%$. In both cases the high mean values of the criteria and their low standard deviations indicate a high quality detection.

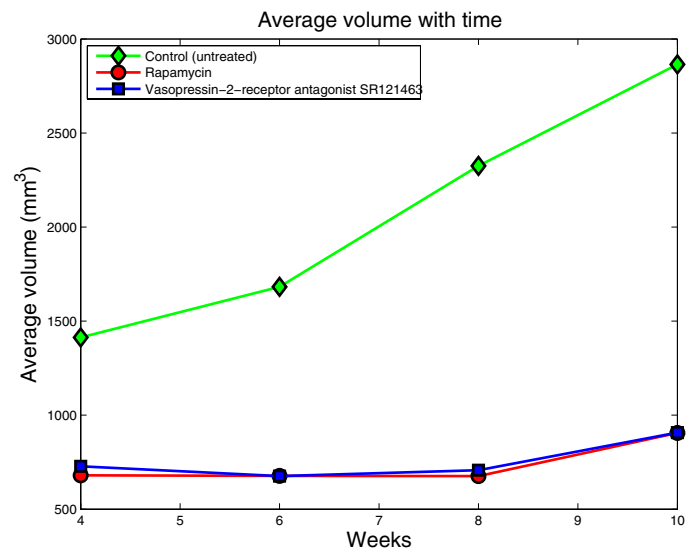

Fig. 2. The average automatically extracted volume of both kidneys over the 48 images as a function of time for the three groups of mice. As expected, the untreated, control, group in green shows a much greater increase in kidney volume. 
In figure 2 are the average values of the automatically extracted volume of both kidneys for the 48 images within every group along the number of weeks after initiation of treatment. As expected, the group of untreated, control, mice shows a large increase in kidney volume. The mice treated with either compound show very limited increase in kidney volume. The processing was performed on an AMD $2 \mathrm{GHz}$ CPU. The average and standard deviation of the computation time were $57 \pm 29 \mathrm{~min}$. They were dominated by the varying time duration of the registrations of the prior shape to the two kidneys.

\section{Discussion}

The spinal cord does not constrain the locations of the kidneys in mice, and thus their relative distance can vary. The kidneys are also connected to the urinary tract and are in contact with fat, the liver, and other organs that can have similar intensities. This necessitates the use of a robust model for mice body anatomy and for the delineation of the kidney boundaries. This is sufficiently represented with the localizations and the registrations of the superspheroids and their use as priors in the graph cuts algorithm. The graph cuts criterion provides segmentations with tight boundaries and penalizes leaking to neighboring anatomic structures.

A manual outlining of the kidneys was performed for thirty images by a medical expert. The enclosed region was used for a volume based validation of the detected kidneys, which demonstrated the high quality of the processing. The surfaces of the detected regions and the manual outlinings could also be compared with measures such as the mean surface distance and the Hausdorff distance. The validation could be repeated with a manual segmentation by a different expert. The parameters of the algorithm were set to accommodate image characteristics such as contrast to noise ratio and resolution. Their settings are also a trade-off between computational requirements and performance. A sensitivity analysis with respect to the parameters of the algorithm would further demonstrate its robustness.

The processing results are in agreement with the effects established with previous techniques, that the two compounds prevent cystogenesis in mice [1]. Previous techniques often involve sacrificing the mice and thus do not allow the monitoring at multiple time points that MRI permits. Micro-CT as well as some MRI acquisition protocols involve the administration of a contrast agent. These agents can cause interfering kidney complications, particularly in mice with compromised kidney function such as those used in this study. The MR acquisitions in this work were performed without the administration of a contrast agent. The processing has been shown to be sufficiently robust to the resulting lower contrast. The method described can analyze MR images of mice in preclinical trials for ADPKD in a non-invasive, reliable, reproducible, and automated manner.

\section{References}

1. Walz, G.: Therapeutic approaches in autosomal dominant polycystic kidney disease (ADPKD): is there light at the end of the tunnel? Nephrol. Dial Transplant. 21(7), 1752-1757 (2006) 
2. Gleason, S., Sarraf, H., Abidi, M., Karakashian, O., Morandi, F.: A new deformable model for analysis of X-ray CT images in preclinical studies of mice for polycystic kidney disease. IEEE Trans. on Medical Imaging 21(10), 1302-1309 (2002)

3. Fei, B., Flask, C., Wang, H., Pi, A., Wilson, D., Shillingford, J., Murcia, N., Weimbs, T., Duerk, J.: Image segmentation, registration and visualization of serial MR images for therapeutic assessment of polycystic kidney disease in transgenic mice. In: Proc. of IEEE-EMBS, pp. 467-469 (2005)

4. Wallace, D., Hou, Y., Huang, Z., Nivens, E., Savinkova, L., Yamaguchi, T., Bilgen, M.: Tracking kidney volume in mice with polycystic kidney disease by magnetic resonance imaging. Kidney International 73, 778-781 (2008)

5. Reichardt, W., Romaker, D., Becker, A., Buechert, M., Walz, G., Elverfeldt, D.: Monitoring kidney and renal cyst volumes applying MR approaches on a rapamycin treated mouse model of ADPKD. In: MAGMA (2008)

6. Abdelmunim, H., Farag, A., Miller, W., AboelGhar, M.: A kidney segmentation approach from DCE-MRI using level sets. In: Proc. of IEEE CVPR Workshops, pp. 1-6 (2008)

7. Ali, A., Farag, A., Baz, A.: Graph cuts framework for kidney segmentation with prior shape constraints. In: Ayache, N., Ourselin, S., Maeder, A. (eds.) MICCAI 2007, Part I. LNCS, vol. 4791, pp. 384-392. Springer, Heidelberg (2007)

8. Gielis, J.: A generic geometric transformation that unifies a wide range of natural and abstract shapes. American Journal of Botany 90(3), 333-338 (2003)

9. Boykov, Y., Lea, G.: Graph cuts and efficient N-D image segmentation. International Journal of Computer Vision 70(2), 109-131 (2006)

10. Gudbjartsson, H., Patz, S.: The Rician distribution of noisy MRI data. Magnetic Resonance in Medicine 34, 910-914 (1995)

11. Hadjidemetriou, S., Studholme, C., Mueller, S., Weiner, M., Schuff, N.: Restoration of MRI data for intensity non-uniformities using local high order intensity statistics. Medical Image Analysis 13(1), 36-48 (2009)

12. Freedman, D., Zhang, T.: Interactive graph cut based segmentation with shape priors. In: Proc. of CVPR, pp. 755-762 (2005)

13. The Insight Segmentation and Registration Toolkit, http://www.itk.org 